Acta Cryst. (2003). C59, 000-000

\title{
Crystal and Molecular Structure of $\left\{\left(\eta^{1} \text { Ind }\right)\left({ }^{t} \operatorname{BuNC}\right) \operatorname{Pd}(\mu-C 1)\right\}_{2}$
}

DAvit ZARGARIAN ${ }^{a}$ AND Christine SUI-SEnG $^{b}$

${ }^{a}$ Département de Chimie, Université de Montréal, C.P. 6128, Succ. Centre-ville, Montréal, Québec, Canada, H3C 3J\%, and ${ }^{b}$ Département de Chimie, Université de Montréal, C.P. 6128,

Succ. Centre-ville, Montréal, Québec, Canada H3C 3J7. E-mail: zargarian.davit@umontreal.ca

\section{Abstract}

Here should be written a short abstract

\section{Comment}

Here should be written the text of the article

\section{Experimental}

Small details about the preparation of the compound.

Crystal data

$\mathrm{C}_{14} \mathrm{H}_{16} \mathrm{ClNPd}$

$M_{r}=340.13$

Monoclinic

$P 2_{1} / c$

$a=6.37760(10) \AA$

$b=13.9174(3) \AA$

$c=16.0809(3) \AA$

$\beta=90.2600(10)^{\circ}$

$V=1427.32(5) \AA^{3}$

$Z=4$

$D_{x}=1.583 \mathrm{Mg} \mathrm{m}^{-3}$

$D_{m}$ not measured

$\mathrm{Cu} K \alpha$ radiation

$\lambda=1.54178 \AA$
Cell parameters from 6374 reflections

$\theta=3.18-72.89^{\circ}$

$\mu=12.018 \mathrm{~mm}^{-1}$

$T=223(2) \mathrm{K}$

Block

Orange

$0.39 \times 0.10 \times 0.08 \mathrm{~mm}$

Crystal source: synthesized by the authors. See text 
Data collection

Bruker AXS Smart 2K/Platform diffractometer

$\omega$ scans

Absorption correction:

multi-scan Sadabs (Sheldrick,1996)

$T_{\min }=0.2720, T_{\max }=0.3820$

17309 measured reflections

2809 independent reflections
2498 reflections with

$$
\begin{aligned}
& \quad>2 \operatorname{sigma}(I) \\
& R_{\text {int }}=0.033 \\
& \theta_{\max }=72.88^{\circ} \\
& h=-7 \rightarrow 6 \\
& k=-17 \rightarrow 16 \\
& l=-19 \rightarrow 19
\end{aligned}
$$

134 standard reflections

\section{every ? reflections}

intensity decay: none

\section{Refinement}

Refinement on $F^{2}$

$R\left[F^{2}>2 \sigma\left(F^{2}\right)\right]=0.0263$

$w R\left(F^{2}\right)=0.0693$

$S=1.018$

2809 reflections

157 parameters

H-atom parameters constrained

$$
\begin{aligned}
& w=1 /\left[\sigma^{2}\left(F_{o}^{2}\right)+(0.0462 P)^{2}+0.0000 P\right] \\
& \text { where } P=\left(F_{o}^{2}+2 F_{c}^{2}\right) / 3 \\
& (\Delta / \sigma)_{\max }=0.001 \\
& \Delta \rho_{\max }=0.356 \mathrm{e}^{-3} \\
& \Delta \rho_{\min }=-0.678 \mathrm{e}^{-3}
\end{aligned}
$$

Extinction correction: none

Scattering factors from International Tables for Crystallography (Vol. C)

Table 1. Selected geometric parameters $\left(\AA,^{\circ}\right)$

$\begin{array}{llll}\mathrm{Pd}-\mathrm{C} 8 & 1.929(3) & \mathrm{C} 3-\mathrm{C} 3 \mathrm{a} & 1.435(4) \\ \mathrm{Pd}-\mathrm{C} 1 & 2.063(3) & \mathrm{C} 3 \mathrm{a}-\mathrm{C} 4 & 1.395(4) \\ \mathrm{Pd}-\mathrm{Cl} & 2.3634(7) & \mathrm{C} 3 \mathrm{a}-\mathrm{C} 7 \mathrm{a} & 1.408(4) \\ \mathrm{Pd}-\mathrm{Cl}^{\mathrm{i}} & 2.4580(7) & \mathrm{C} 4-\mathrm{C} 5 & 1.370(5) \\ \mathrm{Cl}-\mathrm{Pd}{ }^{\mathrm{i}} & 2.4580(7) & \mathrm{C} 5-\mathrm{C} 6 & 1.377(5) \\ \mathrm{N}-\mathrm{C} 8 & 1.138(4) & \mathrm{C} 6-\mathrm{C} 7 & 1.399(5) \\ \mathrm{N}-\mathrm{C} 9 & 1.463(3) & \mathrm{C} 7-\mathrm{C} 7 \mathrm{a} & 1.388(4) \\ \mathrm{C} 1-\mathrm{C} 2 & 1.477(5) & \mathrm{C} 9-\mathrm{C} 10 & 1.509(5) \\ \mathrm{C} 1-\mathrm{C} 7 \mathrm{a} & 1.495(4) & \mathrm{C} 9-\mathrm{C} 11 & 1.510(6) \\ \mathrm{C} 2-\mathrm{C} 3 & 1.349(5) & \mathrm{C} 9-\mathrm{C} 12 & 1.524(5)\end{array}$




\begin{tabular}{|c|c|c|c|}
\hline $\mathrm{C} 8-\mathrm{PD}-\mathrm{C} 1$ & $87.66(12)$ & $\mathrm{C} 7 \mathrm{~A}-\mathrm{C} 3 \mathrm{~A}-\mathrm{C} 3$ & $107.7(3)$ \\
\hline $\mathrm{C} 8-\mathrm{PD}-\mathrm{CL}$ & $177.77(8)$ & $\mathrm{C} 5-\mathrm{C} 4-\mathrm{C} 3 \mathrm{~A}$ & $118.9(3)$ \\
\hline $\mathrm{C} 1-\mathrm{PD}-\mathrm{CL}$ & $90.37(9)$ & $\mathrm{C} 4-\mathrm{C} 5-\mathrm{C} 6$ & $121.2(3)$ \\
\hline $\mathrm{C} 8-\mathrm{PD}-\mathrm{CL}^{\mathrm{i}}$ & $95.22(8)$ & $\mathrm{C} 5-\mathrm{C} 6-\mathrm{C} 7$ & $121.0(3)$ \\
\hline $\mathrm{C} 1-\mathrm{PD}-\mathrm{CL}^{\mathrm{i}}$ & $176.25(8)$ & $\mathrm{C} 7 \mathrm{~A}-\mathrm{C} 7-\mathrm{C} 6$ & $118.5(3)$ \\
\hline $\mathrm{CL}-\mathrm{PD}-\mathrm{CL}^{\mathrm{i}}$ & $86.79(2)$ & $\mathrm{C} 7-\mathrm{C} 7 \mathrm{~A}-\mathrm{C} 3 \mathrm{~A}$ & $120.0(3)$ \\
\hline $\mathrm{PD}-\mathrm{CL}-\mathrm{PD}^{\mathrm{i}}$ & $93.21(2)$ & $\mathrm{C} 7-\mathrm{C} 7 \mathrm{~A}-\mathrm{C} 1$ & $131.3(3)$ \\
\hline $\mathrm{C} 8-\mathrm{N}-\mathrm{C} 9$ & $171.3(3)$ & $\mathrm{C} 3 \mathrm{~A}-\mathrm{C} 7 \mathrm{~A}-\mathrm{C} 1$ & $108.7(3)$ \\
\hline $\mathrm{C} 2-\mathrm{C} 1-\mathrm{C} 7 \mathrm{~A}$ & $103.0(2)$ & $\mathrm{N}-\mathrm{C} 8-\mathrm{PD}$ & $179.3(3)$ \\
\hline $\mathrm{C} 2-\mathrm{C} 1-\mathrm{PD}$ & $105.6(2)$ & $\mathrm{N}-\mathrm{C} 9-\mathrm{C} 10$ & $106.6(3)$ \\
\hline $\mathrm{C} 7 \mathrm{~A}-\mathrm{C} 1-\mathrm{PD}$ & $118.41(19)$ & $\mathrm{N}-\mathrm{C} 9-\mathrm{C} 11$ & $106.2(3)$ \\
\hline $\mathrm{C} 3-\mathrm{C} 2-\mathrm{C} 1$ & $110.5(3)$ & $\mathrm{C} 10-\mathrm{C} 9-\mathrm{C} 11$ & $113.1(4)$ \\
\hline $\mathrm{C} 2-\mathrm{C} 3-\mathrm{C} 3 \mathrm{~A}$ & $110.0(3)$ & $\mathrm{N}-\mathrm{C} 9-\mathrm{C} 12$ & $108.1(3)$ \\
\hline $\mathrm{C} 4-\mathrm{C} 3 \mathrm{~A}-\mathrm{C} 7 \mathrm{~A}$ & $120.4(3)$ & $\mathrm{C} 10-\mathrm{C} 9-\mathrm{C} 12$ & $111.3(4)$ \\
\hline $\begin{array}{l}\mathrm{C} 4-\mathrm{C} 3 \mathrm{~A}-\mathrm{C} 3 \\
\mathrm{C} 1-\mathrm{PD}-\mathrm{CL}-\mathrm{PD}^{\mathrm{i}}\end{array}$ & $\begin{array}{c}131.9(3) \\
-177.54(9)\end{array}$ & $\begin{array}{l}\mathrm{C} 11-\mathrm{C} 9-\mathrm{C} 12 \\
\mathrm{C} 4-\mathrm{C} 5-\mathrm{C} 6-\mathrm{C} 7\end{array}$ & $\begin{array}{r}111.2(3) \\
1.2(5)\end{array}$ \\
\hline $\mathrm{CL}^{\mathrm{i}}-\mathrm{PD}-\mathrm{CL}-\mathrm{PD}^{\mathrm{i}}$ & 0 & $\mathrm{C} 5-\mathrm{C} 6-\mathrm{C} 7-\mathrm{C} 7 \mathrm{~A}$ & $-0.7(5)$ \\
\hline $\mathrm{C} 8-\mathrm{PD}-\mathrm{C} 1-\mathrm{C} 2$ & $-75.9(2)$ & $\mathrm{C} 6-\mathrm{C} 7-\mathrm{C} 7 \mathrm{~A}-\mathrm{C} 3 \mathrm{~A}$ & $-0.2(4)$ \\
\hline $\mathrm{CL}-\mathrm{PD}-\mathrm{C} 1-\mathrm{C} 2$ & $105.2(2)$ & $\mathrm{C} 6-\mathrm{C} 7-\mathrm{C} 7 \mathrm{~A}-\mathrm{C} 1$ & $177.6(3)$ \\
\hline $\mathrm{C} 8-\mathrm{PD}-\mathrm{C} 1-\mathrm{C} 7 \mathrm{~A}$ & $38.8(2)$ & $\mathrm{C} 4-\mathrm{C} 3 \mathrm{~A}-\mathrm{C} 7 \mathrm{~A}-\mathrm{C} 7$ & $0.7(4)$ \\
\hline $\mathrm{C} 7 \mathrm{~A}-\mathrm{C} 1-\mathrm{C} 2-\mathrm{C} 3$ & $-1.5(4)$ & $\mathrm{C} 3-\mathrm{C} 3 \mathrm{~A}-\mathrm{C} 7 \mathrm{~A}-\mathrm{C} 7$ & $179.7(3)$ \\
\hline $\mathrm{PD}-\mathrm{C} 1-\mathrm{C} 2-\mathrm{C} 3$ & $123.4(3)$ & $\mathrm{C} 4-\mathrm{C} 3 \mathrm{~A}-\mathrm{C} 7 \mathrm{~A}-\mathrm{C} 1$ & $-177.5(3)$ \\
\hline $\mathrm{C} 1-\mathrm{C} 2-\mathrm{C} 3-\mathrm{C} 3 \mathrm{~A}$ & $2.5(4)$ & $\mathrm{C} 3-\mathrm{C} 3 \mathrm{~A}-\mathrm{C} 7 \mathrm{~A}-\mathrm{C} 1$ & $1.4(3)$ \\
\hline $\mathrm{C} 2-\mathrm{C} 3-\mathrm{C} 3 \mathrm{~A}-\mathrm{C} 4$ & $176.4(3)$ & $\mathrm{C} 2-\mathrm{C} 1-\mathrm{C} 7 \mathrm{~A}-\mathrm{C} 7$ & $-178.0(3)$ \\
\hline $\mathrm{C} 2-\mathrm{C} 3-\mathrm{C} 3 \mathrm{~A}-\mathrm{C} 7 \mathrm{~A}$ & $-2.5(4)$ & $\mathrm{PD}-\mathrm{C} 1-\mathrm{C} 7 \mathrm{~A}-\mathrm{C} 7$ & $65.9(4)$ \\
\hline $\mathrm{C} 7 \mathrm{~A}-\mathrm{C} 3 \mathrm{~A}-\mathrm{C} 4-\mathrm{C} 5$ & $-0.3(5)$ & $\mathrm{C} 2-\mathrm{C} 1-\mathrm{C} 7 \mathrm{~A}-\mathrm{C} 3 \mathrm{~A}$ & $0.0(3)$ \\
\hline $\mathrm{C} 3-\mathrm{C} 3 \mathrm{~A}-\mathrm{C} 4-\mathrm{C} 5$ & $-179.0(3)$ & $\mathrm{PD}-\mathrm{C} 1-\mathrm{C} 7 \mathrm{~A}-\mathrm{C} 3 \mathrm{~A}$ & $-116.1(2)$ \\
\hline $\mathrm{C} 3 \mathrm{~A}-\mathrm{C} 4-\mathrm{C} 5-\mathrm{C} 6$ & $-0.7(5)$ & & \\
\hline
\end{tabular}

Symmetry codes: (i) $1-x, 1-y, 1-z$.

The structure was solved by direct method with ShelxS-97. All non-H atoms were refined by full-matrix least-squares with anisotropic displacement parameters while $\mathrm{H}$ atoms were placed in idealized position.

Data collection: SAINT (Bruker, 1999). Cell refinement: SMART (Bruker, 1999). Data reduction: SAINT (Bruker, 1999). Program(s) used to solve structure: SHELXS97 (Sheldrick, 1997). Program(s) used to refine structure: SHELXL97 (Sheldrick, 1997). Molecular graphics: XP (Bruker, 1999). Software used to prepare material for publication: UdMX (local program).

We are grateful to the Natural Sciences and Engineering Research Council of Canada and the Ministère de l'Education du Québec for financial support. 
$\overline{\text { Supplementary data for this paper are available from the IUCr electronic archives (Reference: ). }}$ Services for accessing these data are described at the back of the journal.

\section{References}

Bruker (1997). SHELXTL Release 5.10; The Complete Software Package for Single Crystal Structure Determination, Bruker AXS Inc., Madison,USA.

Bruker (1999). SAINT Release 6.06; Integration Software for Single Crystal Data, Bruker AXS Inc., Madison,USA.

Bruker (1999). SMART Release 5.059; Bruker Molecular Analysis Research Tool, Bruker AXS Inc., Madison, USA.

Sheldrick, G. M. (1996). SADABS, Bruker Area Detector Absorption Corrections, Bruker AXS Inc., Madison, USA.

Sheldrick, G. M. (1997). SHELXS97. Program for the Solution of Crystal Structures. University of Gottingen, Germany.

Sheldrick, G. M. (1997). SHELXL97. Program for the Refinement of Crystal Structures. University of Gottingen, Germany.

Spek, A. L. (1995). PLATON, July 1995 version; Molecular Geometry Program, University of Utrecht, The Netherlands.;

Fig 1 ORTEP view of the title compound. Thermal ellipsoids are shown at 30\% probability levels.

Table 1. Selected geometric parameters $\left(\AA{ }^{\circ}\right)$ for the title compound. 


\section{Supplementary data}

The tables of data shown below are not normally printed in Acta Cryst. Section $C$ but the data will be available electronically via the online contents pages at

http://journals.iucr.org/c/journalhomepage.html

Table S1. Fractional atomic coordinates and equivalent isotropic displacement parameters $\left(\AA^{2}\right)$

\begin{tabular}{|c|c|c|c|c|}
\hline & $x$ & $y$ & $z$ & $U_{\text {eq }}$ \\
\hline $\mathrm{Pd}$ & $0.35001(3)$ & $\mathbf{0 . 5 6 9 6 5 0}(13)$ & 0.431191 & $0.03058(9)$ \\
\hline $\mathrm{Cl}$ & $0.42183(13)$ & 0.40408 & $0.44732(5)$ & $0.04600(19)$ \\
\hline $\mathrm{N}$ & $0.2553(4)$ & $0.78320(17)$ & $0.40267(15)$ & $0.0412(6)$ \\
\hline $\mathrm{C} 1$ & $0.1505(5)$ & $0.5399(2)$ & $0.33320(18)$ & $0.0387(6)$ \\
\hline $\mathrm{H} 1$ & 0.1705 & 0.4726 & 0.3149 & 0.046 \\
\hline $\mathrm{C} 2$ & $-0.0634(5)$ & $0.5523(3)$ & $0.3662(2)$ & $0.0499(8)$ \\
\hline $\mathrm{H} 2$ & -0.1173 & 0.5196 & 0.4126 & 0.060 \\
\hline C3 & $-0.1709(5)$ & $0.6173(3)$ & $0.3206(2)$ & $0.0503(8)$ \\
\hline H3 & -0.3082 & 0.6380 & 0.3317 & 0.060 \\
\hline C3A & $-0.0447(5)$ & $0.6500(2)$ & $0.25258(18)$ & $0.0387(6)$ \\
\hline $\mathrm{C} 4$ & $-0.0873(5)$ & $0.7123(2)$ & $0.1867(2)$ & $0.0505(8)$ \\
\hline $\mathrm{H} 4$ & -0.2191 & 0.7420 & 0.1818 & 0.061 \\
\hline C5 & $0.0660(6)$ & $0.7294(3)$ & $0.1291(2)$ & $0.0560(9)$ \\
\hline H5 & 0.0379 & 0.7707 & 0.0842 & 0.067 \\
\hline C6 & $0.2606(6)$ & $0.6871(3)$ & $0.1359(2)$ & $0.0550(9)$ \\
\hline H6 & 0.3638 & 0.7009 & 0.0961 & 0.066 \\
\hline $\mathrm{C} 7$ & $0.3069(5)$ & $0.6238(2)$ & $0.20104(18)$ & $0.0442(7)$ \\
\hline $\mathrm{H} 7$ & 0.4393 & 0.5945 & 0.2051 & 0.053 \\
\hline $\mathrm{C} 7 \mathrm{~A}$ & $0.1527(4)$ & $0.6053(2)$ & $0.25949(17)$ & $0.0341(6)$ \\
\hline $\mathrm{C} 8$ & $0.2893(5)$ & $0.7039(2)$ & $0.41349(17)$ & $0.0365(6)$ \\
\hline C9 & $0.2050(6)$ & $0.8810(2)$ & $0.3758(2)$ & 0.0541 (9) \\
\hline $\mathrm{C} 10$ & $0.2965(8)$ & $0.8927(3)$ & $0.2900(2)$ & $0.0776(14)$ \\
\hline H10A & 0.4468 & 0.8823 & 0.2924 & 0.116 \\
\hline H10B & 0.2682 & 0.9571 & 0.2698 & 0.116 \\
\hline $\mathrm{H} 10 \mathrm{C}$ & 0.2334 & 0.8461 & 0.2526 & 0.116 \\
\hline C11 & $-0.0313(8)$ & $0.8885(3)$ & $0.3768(3)$ & $0.0880(15)$ \\
\hline H11A & -0.0907 & 0.8419 & 0.3386 & 0.132 \\
\hline H11B & -0.0730 & 0.9527 & 0.3600 & 0.132 \\
\hline $\mathrm{H} 11 \mathrm{C}$ & -0.0818 & 0.8759 & 0.4325 & 0.132 \\
\hline $\mathrm{C} 12$ & $0.3046(8)$ & $0.9507(3)$ & $0.4374(2)$ & $0.0767(14)$ \\
\hline $\mathrm{H} 12 \mathrm{~A}$ & 0.2395 & 0.9431 & 0.4914 & 0.115 \\
\hline $\mathrm{H} 12 \mathrm{~B}$ & 0.2842 & 1.0161 & 0.4180 & 0.115 \\
\hline $\mathrm{H} 12 \mathrm{C}$ & 0.4535 & 0.9374 & 0.4420 & 0.115 \\
\hline
\end{tabular}

Table S2. Anisotropic displacement parameters $\left(\AA^{2}\right)$

\begin{tabular}{|c|c|c|c|c|c|c|}
\hline & $U_{11}$ & $U_{22}$ & $U_{33}$ & $U_{12}$ & $U_{13}$ & $U_{23}$ \\
\hline $\mathrm{Pd}$ & $0.04060(14)$ & $0.02281(12)$ & $0.02829(12)$ & $0.00270(7)$ & $-0.00536(8)$ & $0.00076(7)$ \\
\hline $\mathrm{Cl}$ & $0.0687(5)$ & $0.0238(3)$ & $0.0454(4)$ & $0.0054(3)$ & $-0.0230(3)$ & $-0.0031(3)$ \\
\hline $\mathrm{N}$ & $0.0599(16)$ & $0.0257(12)$ & $0.0380(14)$ & $0.0043(11)$ & $-0.0139(11)$ & $-0.0002(10)$ \\
\hline C1 & $0.0504(18)$ & $0.0324(14)$ & $0.0333(15)$ & $-0.0043(12)$ & $-0.0118(12)$ & $-0.0006(12)$ \\
\hline $\mathrm{C} 2$ & $0.050(2)$ & $0.058(2)$ & $0.0423(18)$ & $-0.0206(15)$ & $-0.0056(14)$ & $0.0067(15)$ \\
\hline C3 & $0.0354(18)$ & $0.065(2)$ & $0.050(2)$ & $-0.0032(14)$ & $-0.0040(13)$ & $-0.0054(16)$ \\
\hline $\mathrm{C} 3 \mathrm{~A}$ & $0.0428(16)$ & $0.0370(15)$ & $0.0364(15)$ & $-0.0037(12)$ & $-0.0097(11)$ & $-0.0036(12)$ \\
\hline 4 & $0.060(2)$ & $0.0435(18)$ & $0.0476(19)$ & $0.0064(15)$ & $-0.0245(15)$ & $0.0010(14)$ \\
\hline & $0.086(3)$ & $0.0480(19)$ & $0.0339(17)$ & $-0.0054(18)$ & $-0.0176(16)$ & $0.0057(14)$ \\
\hline C6 & $0.075(2)$ & $0.059(2)$ & $0.0313(16)$ & $-0.0167(18)$ & $0.0031(15)$ & $-0.0019(15)$ \\
\hline 7 & $0.0515(19)$ & $0.0477(18)$ & $0.0333(15)$ & $-0.0035(14)$ & $-0.0008(12)$ & $-0.0105(13)$ \\
\hline $7 \mathrm{~A}$ & $0.0420(16)$ & $0.0291(14)$ & $0.0313(14)$ & $-0.0013(11)$ & $-0.0082(11)$ & $-0.0050(11)$ \\
\hline & $0.0436(16)$ & $0.0355(16)$ & $0.0303(14)$ & $-0.0003(12)$ & $-0.0067(11)$ & $-0.0023(11)$ \\
\hline & $0.092(3)$ & $0.0232(14)$ & $0.0467(19)$ & $0.0113(15)$ & $-0.0206(17)$ & $-0.0008(13)$ \\
\hline
\end{tabular}




\begin{tabular}{|c|c|c|c|c|c|c|}
\hline $\mathrm{C} 10$ & $0.142(4)$ & $0.040(2)$ & $0.050(2)$ & $-0.012(2)$ & $-0.018(2)$ & $0.0083(17)$ \\
\hline $\mathrm{C} 11$ & $0.099(4)$ & $0.055(3)$ & $0.110(4)$ & $0.036(2)$ & $-0.032(3)$ & $-0.005(3)$ \\
\hline $\mathrm{C} 12$ & 0.139 (4) & $0.0330(18)$ & $0.058(3)$ & $-0.002(2)$ & $-0.032(3)$ & $-0.0065(16)$ \\
\hline
\end{tabular}

$\mathrm{Pd}-\mathrm{C} 8$

$\mathrm{Pd}-\mathrm{C} 1$

$\mathrm{Pd}-\mathrm{Cl}$

$\mathrm{Pd}-\mathrm{Cl}^{\mathrm{i}}$

$\mathrm{Cl}-\mathrm{Pd}^{\mathrm{i}}$

$\mathrm{N}-\mathrm{C} 8$

$\mathrm{N}-\mathrm{C} 9$

$\mathrm{C} 1-\mathrm{C} 2$

C1- C7a

$\mathrm{C} 1-\mathrm{H} 1$

$\mathrm{C} 2-\mathrm{C} 3$

$\mathrm{C} 2-\mathrm{H} 2$

C3-C3a

$\mathrm{C} 3-\mathrm{H} 3$

$\mathrm{C} 3 \mathrm{a}-\mathrm{C} 4$

$\mathrm{C} 3 \mathrm{a}-\mathrm{C} 7 \mathrm{a}$

$\mathrm{C} 4-\mathrm{C} 5$

$\mathrm{C} 4-\mathrm{H} 4$

$\mathrm{C} 8-\mathrm{PD}-\mathrm{C}$

$\mathrm{C} 8-\mathrm{PD}-\mathrm{CL}$

$\mathrm{C} 1-\mathrm{PD}-\mathrm{CL}$

$\mathrm{C} 8-\mathrm{PD}-\mathrm{CL}^{\mathrm{i}}$

$\mathrm{C} 1-\mathrm{PD}-\mathrm{CL}^{1}$

$\mathrm{CL}-\mathrm{PD}-\mathrm{CL}^{\mathrm{i}}$

$\mathrm{PD}-\mathrm{CL}-\mathrm{PD}^{\mathrm{i}}$

$\mathrm{C} 8-\mathrm{N}-\mathrm{C} 9$

$\mathrm{C} 2-\mathrm{C} 1-\mathrm{C} 7 \mathrm{~A}$

$\mathrm{C} 2-\mathrm{C} 1-\mathrm{PD}$

$\mathrm{C} 7 \mathrm{~A}-\mathrm{C} 1-\mathrm{PD}$

$\mathrm{C} 2-\mathrm{C} 1-\mathrm{H} 1$

$\mathrm{C} 7 \mathrm{~A}-\mathrm{C} 1-\mathrm{H} 1$

$\mathrm{PD}-\mathrm{C} 1-\mathrm{H} 1$

C3 $-\mathrm{C} 2-\mathrm{C} 1$

C3 $-\mathrm{C} 2-\mathrm{H} 2$

$\mathrm{C} 1-\mathrm{C} 2-\mathrm{H} 2$

$\mathrm{C} 2-\mathrm{C} 3-\mathrm{C} 3 \mathrm{~A}$

$\mathrm{C} 2-\mathrm{C} 3-\mathrm{H} 3$

$\mathrm{C} 3 \mathrm{~A}-\mathrm{C} 3-\mathrm{H} 3$

$\mathrm{C} 4-\mathrm{C} 3 \mathrm{~A}-\mathrm{C} 7 \mathrm{~A}$

$\mathrm{C} 4-\mathrm{C} 3 \mathrm{~A}-\mathrm{C} 3$

$\mathrm{C} 7 \mathrm{~A}-\mathrm{C} 3 \mathrm{~A}-\mathrm{C} 3$

$\mathrm{C} 5-\mathrm{C} 4-\mathrm{C} 3 \mathrm{~A}$

$\mathrm{C} 5-\mathrm{C} 4-\mathrm{H} 4$

$\mathrm{C} 3 \mathrm{~A}-\mathrm{C} 4-\mathrm{H} 4$

$\mathrm{C} 4-\mathrm{C} 5-\mathrm{C} 6$

$\mathrm{C} 4-\mathrm{C} 5-\mathrm{H} 5$

C6- $55-\mathrm{H} 5$

$\mathrm{C} 5-\mathrm{C} 6-\mathrm{C} 7$

$\mathrm{C} 5-\mathrm{C} 6-\mathrm{H} 6$

$\mathrm{C} 7-\mathrm{C} 6-\mathrm{H} 6$

Table S3. Geometric parameters $\left(\AA,^{\circ}\right)$

\begin{tabular}{|c|c|c|}
\hline $1.929(3)$ & $\mathrm{C} 5-\mathrm{C} 6$ & $1.377(5)$ \\
\hline $2.063(3)$ & $\mathrm{C} 5-\mathrm{H} 5$ & 0.94 \\
\hline $2.3634(7)$ & $\mathrm{C} 6-\mathrm{C} 7$ & $1.399(5)$ \\
\hline $2.4580(7)$ & $\mathrm{C} 6-\mathrm{H} 6$ & 0.94 \\
\hline $2.4580(7)$ & $\mathrm{C} 7-\mathrm{C} 7 \mathrm{a}$ & $1.388(4)$ \\
\hline $1.138(4)$ & $\mathrm{C} 7-\mathrm{H} 7$ & 0.94 \\
\hline $1.463(3)$ & $\mathrm{C} 9-\mathrm{C} 10$ & $1.509(5)$ \\
\hline $1.477(5)$ & $\mathrm{C} 9-\mathrm{C} 11$ & $1.510(6)$ \\
\hline $1.495(4)$ & $\mathrm{C} 9-\mathrm{C} 12$ & $1.524(5)$ \\
\hline 0.99 & $\mathrm{C} 10-\mathrm{H} 10 \mathrm{a}$ & 0.97 \\
\hline $1.349(5)$ & $\mathrm{C} 10-\mathrm{H} 10 b$ & 0.97 \\
\hline 0.94 & $\mathrm{C} 10-\mathrm{H} 10 c$ & 0.97 \\
\hline $1.435(4)$ & $\mathrm{C} 11-\mathrm{H} 11 \mathrm{a}$ & 0.97 \\
\hline 0.94 & $\mathrm{C} 11-\mathrm{H} 11 b$ & 0.97 \\
\hline $1.395(4)$ & $\mathrm{C} 11-\mathrm{H} 11 c$ & 0.97 \\
\hline $1.408(4)$ & $\mathrm{C} 12-\mathrm{H} 12 \mathrm{a}$ & 0.97 \\
\hline $1.370(5)$ & $\mathrm{C} 12-\mathrm{H} 12 b$ & 0.97 \\
\hline 0.94 & $\mathrm{C} 12-\mathrm{H} 12 c$ & 0.97 \\
\hline $87.66(12)$ & $\mathrm{C} 7 \mathrm{~A}-\mathrm{C} 7-\mathrm{C} 6$ & $118.5(3)$ \\
\hline $177.77(8)$ & $\mathrm{C} 7 \mathrm{~A}-\mathrm{C} 7-\mathrm{H} 7$ & 120.8 \\
\hline $90.37(9)$ & $\mathrm{C} 6-\mathrm{C} 7-\mathrm{H} 7$ & 120.8 \\
\hline $95.22(8)$ & $\mathrm{C} 7-\mathrm{C} 7 \mathrm{~A}-\mathrm{C} 3 \mathrm{~A}$ & $120.0(3)$ \\
\hline $176.25(8)$ & $\mathrm{C} 7-\mathrm{C} 7 \mathrm{~A}-\mathrm{C} 1$ & $131.3(3)$ \\
\hline $86.79(2)$ & $\mathrm{C} 3 \mathrm{~A}-\mathrm{C} 7 \mathrm{~A}-\mathrm{C} 1$ & $108.7(3)$ \\
\hline $93.21(2)$ & $\mathrm{N}-\mathrm{C} 8-\mathrm{PD}$ & $179.3(3)$ \\
\hline $171.3(3)$ & $\mathrm{N}-\mathrm{C} 9-\mathrm{C} 10$ & $106.6(3)$ \\
\hline $103.0(2)$ & $\mathrm{N}-\mathrm{C} 9-\mathrm{C} 11$ & $106.2(3)$ \\
\hline $105.6(2)$ & $\mathrm{C} 10-\mathrm{C} 9-\mathrm{C} 11$ & $113.1(4)$ \\
\hline $118.41(19)$ & $\mathrm{N}-\mathrm{C} 9-\mathrm{C} 12$ & $108.1(3)$ \\
\hline 109.8 & $\mathrm{C} 10-\mathrm{C} 9-\mathrm{C} 12$ & $111.3(4)$ \\
\hline 109.8 & $\mathrm{C} 11-\mathrm{C} 9-\mathrm{C} 12$ & $111.2(3)$ \\
\hline 109.8 & $\mathrm{C} 9-\mathrm{C} 10-\mathrm{H} 10 \mathrm{~A}$ & 109.5 \\
\hline $110.5(3)$ & $\mathrm{C} 9-\mathrm{C} 10-\mathrm{H} 10 \mathrm{~B}$ & 109.5 \\
\hline 124.7 & $\mathrm{H} 10 \mathrm{~A}-\mathrm{C} 10-\mathrm{H} 10 \mathrm{~B}$ & 109.5 \\
\hline 124.7 & $\mathrm{C} 9-\mathrm{C} 10-\mathrm{H} 10 \mathrm{C}$ & 109.5 \\
\hline $110.0(3)$ & $\mathrm{H} 10 \mathrm{~A}-\mathrm{C} 10-\mathrm{H} 10 \mathrm{C}$ & 109.5 \\
\hline 125 & $\mathrm{H} 10 \mathrm{~B}-\mathrm{C} 10-\mathrm{H} 10 \mathrm{C}$ & 109.5 \\
\hline 125 & $\mathrm{C} 9-\mathrm{C} 11-\mathrm{H} 11 \mathrm{~A}$ & 109.5 \\
\hline $120.4(3)$ & $\mathrm{C} 9-\mathrm{C} 11-\mathrm{H} 11 \mathrm{~B}$ & 109.5 \\
\hline $131.9(3)$ & $\mathrm{H} 11 \mathrm{~A}-\mathrm{C} 11-\mathrm{H} 11 \mathrm{~B}$ & 109.5 \\
\hline $107.7(3)$ & $\mathrm{C} 9-\mathrm{C} 11-\mathrm{H} 11 \mathrm{C}$ & 109.5 \\
\hline $118.9(3)$ & $\mathrm{H} 11 \mathrm{~A}-\mathrm{C} 11-\mathrm{H} 11 \mathrm{C}$ & 109.5 \\
\hline 120.5 & $\mathrm{H} 11 \mathrm{~B}-\mathrm{C} 11-\mathrm{H} 11 \mathrm{C}$ & 109.5 \\
\hline 120.5 & $\mathrm{C} 9-\mathrm{C} 12-\mathrm{H} 12 \mathrm{~A}$ & 109.5 \\
\hline $121.2(3)$ & $\mathrm{C} 9-\mathrm{C} 12-\mathrm{H} 12 \mathrm{~B}$ & 109.5 \\
\hline 119.4 & $\mathrm{H} 12 \mathrm{~A}-\mathrm{C} 12-\mathrm{H} 12 \mathrm{~B}$ & 109.5 \\
\hline 119.4 & $\mathrm{C} 9-\mathrm{C} 12-\mathrm{H} 12 \mathrm{C}$ & 109.5 \\
\hline $121.0(3)$ & $\mathrm{H} 12 \mathrm{~A}-\mathrm{C} 12-\mathrm{H} 12 \mathrm{C}$ & 109.5 \\
\hline 119.5 & $\mathrm{H} 12 \mathrm{~B}-\mathrm{C} 12-\mathrm{H} 12 \mathrm{C}$ & 109.5 \\
\hline 119.5 & & \\
\hline
\end{tabular}




$\begin{array}{lrlr}\mathrm{C} 1-\mathrm{PD}-\mathrm{CL}-\mathrm{PD}^{\mathrm{i}} & -177.54(9) & \mathrm{C} 4-\mathrm{C} 5-\mathrm{C} 6-\mathrm{C} 7 & 1.2(5) \\ \mathrm{CL}-\mathrm{PD}-\mathrm{CL}-\mathrm{PD}^{\mathrm{i}} & 0 & \mathrm{C} 5-\mathrm{C} 6-\mathrm{C} 7-\mathrm{C} 7 \mathrm{~A} & -0.7(5) \\ \mathrm{C} 8-\mathrm{PD}-\mathrm{C} 1-\mathrm{C} 2 & -75.9(2) & \mathrm{C} 6-\mathrm{C} 7-\mathrm{C} 7 \mathrm{~A}-\mathrm{C} 3 \mathrm{~A} & -0.2(4) \\ \mathrm{CL}-\mathrm{PD}-\mathrm{C} 1-\mathrm{C} 2 & 105.2(2) & \mathrm{C} 6-\mathrm{C} 7-\mathrm{C} 7 \mathrm{~A}-\mathrm{C} 1 & 177.6(3) \\ \mathrm{C} 8-\mathrm{PD}-\mathrm{C} 1-\mathrm{C} 7 \mathrm{~A} & 38.8(2) & \mathrm{C} 4-\mathrm{C} 3 \mathrm{~A}-\mathrm{C} 7 \mathrm{~A}-\mathrm{C} 7 & 0.7(4) \\ \mathrm{C} 7 \mathrm{~A}-\mathrm{C} 1-\mathrm{C} 2-\mathrm{C} 3 & -1.5(4) & \mathrm{C} 4-\mathrm{C} 3 \mathrm{~A}-\mathrm{C} 7 \mathrm{~A}-\mathrm{C} 7 & 179.7(3) \\ \mathrm{PD}-\mathrm{C} 1-\mathrm{C} 2-\mathrm{C} 3 & 123.4(3) & \mathrm{C} 3-\mathrm{C} 3 \mathrm{~A}-\mathrm{C} 7 \mathrm{~A}-\mathrm{C} 1 & -177.5(3) \\ \mathrm{C} 1-\mathrm{C} 2-\mathrm{C} 3-\mathrm{C} 3 \mathrm{~A} & 2.5(4) & \mathrm{C} 2-\mathrm{C} 1-\mathrm{C} 7 \mathrm{~A}-\mathrm{C} 7 & 1.4(3) \\ \mathrm{C} 2-\mathrm{C} 3-\mathrm{C} 3 \mathrm{~A}-\mathrm{C} 4 & \mathrm{PD}-\mathrm{C} 1-\mathrm{C} 7 \mathrm{~A}-\mathrm{C} 7 & -178.0(3) \\ \mathrm{C} 2-\mathrm{C} 3-\mathrm{C} 3 \mathrm{~A}-\mathrm{C} 7 \mathrm{~A} & -2.5(4) & \mathrm{C} 2-\mathrm{C} 1-\mathrm{C} 7 \mathrm{~A}-\mathrm{C} 3 \mathrm{~A} & 65.9(4) \\ \mathrm{C} 7 \mathrm{~A}-\mathrm{C} 3 \mathrm{~A}-\mathrm{C} 4-\mathrm{C} 5 & -0.3(5) & \mathrm{PD}-\mathrm{C} 1-\mathrm{C} 7 \mathrm{~A}-\mathrm{C} 3 \mathrm{~A} & 0.0(3) \\ \mathrm{C} 3-\mathrm{C} 3 \mathrm{~A}-\mathrm{C} 4-\mathrm{C} 5 & -179.0(3) & & -116.1(2)\end{array}$

$\mathrm{C} 3 \mathrm{~A}-\mathrm{C} 4-\mathrm{C} 5-\mathrm{C} 6$

$-0.7(5)$

Symmetry codes: (i) $1-x, 1-y, 1-z$. 